Canadian

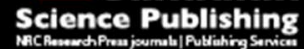

Canadian Journal of Chemistry Revue canadienne de chimie

\title{
Using the Localized Pair Model to Describe the Nature of the Hydrogen Bond
}

\begin{tabular}{|c|c|}
\hline Journal: & Canadian Journal of Chemistry \\
\hline Manuscript ID & cjc-2017-0677.R1 \\
\hline Manuscript Type: & Article \\
\hline Date Submitted by the Author: & 18-Jan-2018 \\
\hline Complete List of Authors: & $\begin{array}{l}\text { Proud, Adam; University of Prince Edward Island, Chemistry } \\
\text { Sheppard, Brendan; University of Prince Edward Island, Chemistry } \\
\text { Pearson, Jason; University of Prince Edward Island, }\end{array}$ \\
\hline $\begin{array}{l}\text { Is the invited manuscript for } \\
\text { consideration in a Special } \\
\text { Issue?: }\end{array}$ & Dalhousie \\
\hline Keyword: & $\begin{array}{l}\text { Hydrogen Bonding, Density Functional Theory, Electron Density, Extracule } \\
\text { Density, Single Electron Pair Distribution Analysis }\end{array}$ \\
\hline
\end{tabular}

SCHOLARONE $^{\text {m }}$

Manuscripts 


\title{
Using the Localized Pair Model to Describe the Nature of the Hydrogen Bond
}

\author{
Adam J. Proud, Brendan J.H. Sheppard, and Jason K. Pearson* \\ Department of Chemistry, University of Prince Edward Island, \\ Charlottetown, PE, Canada, C1A 4 P3
}

(Dated: January 17, 2018)

\begin{abstract}
Herein, we present a novel study of hydrogen bonding using the localized pair model. Using localized molecular orbitals, we examine how the two localized electron pairs involved in hydrogen bonding change upon the formation of the interaction, as well as while the distance between the two species within the complex change. This is achieved through the use of extracule densities, which describe the probability associated with the centre-of-mass of an electron pair. Quantitative assessments are performed by analyzing the extracule density demonstrating that this method is on par with other density-based tools for the analysis of hydrogen bonds and their strengths.
\end{abstract}




\section{INTRODUCTION}

$\therefore$... under certain conditions an atom of hydrogen is attracted by rather strong forces to two atoms, instead of only one, so that it may be considered to be acting as a bond between them. This is called the hydrogen bond.' - Linus Pauling[1]

While our understanding of the hydrogen bond has improved significantly since it was defined by Pauling, his original definition does accurately describe the unique interaction. A hydrogen atom, while covalently bound to one electronegative atom, may interact with a second atom within a separate molecule or a separate moiety within the same molecule. The strength of this interaction depends on various factors including molecular geometries and electrostatics but can vary from $0.5-45 \mathrm{kcal} / \mathrm{mol}$.[2]

Hydrogen bonding is highly prevalent in nature through its extensive involvement in the secondary structure of proteins, the stabilization of the double helix structure of DNA, and in many of the remarkable properties of $\mathrm{H}_{2} \mathrm{O}$. It was first described in the 1920s and its definition continues to change as our understanding of the interaction improves. [3] While it was once largely considered an electrostatic interaction, recently a resonance-covalency model has begun to emerge as a more complete characterization. [2, 3]

As hydrogen bonding involves the through-space interaction of a lone pair of electrons of a heavy atom $(\mathrm{Y})$ with an electron deficient neighbouring hydrogen $(\mathrm{H}-\mathrm{X})$, observing the changes in electron positions and electron pair separations represent intuitive ways to analyze and further characterize hydrogen bonding interactions. While the wavefunction contains all necessary information regarding the positions of all $N$ electrons for this purpose, dealing with such vast amounts of information is not generally tractable. However, as noted, hydrogen bonds primarily involve interactions between two localized sets of electron pairs: those involved in the covalent $\mathrm{X}-\mathrm{H}$ bond, $\sigma_{X H}$, of the hydrogen bond donor and those in the lone pair of atom $\mathrm{Y}, n_{Y}$, of the hydrogen bond acceptor. Thus, this interaction can be simplified by analyzing individual electron pairs. To do so, we can simplify the wavefunction, $\Psi\left(\mathbf{x}_{1}, \mathbf{x}_{2}, \ldots, \mathbf{x}_{\mathrm{N}}\right)$, by averaging over all but two electronic coordinates as

$$
\rho\left(\mathbf{r}_{1}, \mathbf{r}_{\mathbf{2}}\right)=\int\left|\Psi\left(\mathbf{x}_{\mathbf{1}}, \mathbf{x}_{\mathbf{2}}, \ldots, \mathbf{x}_{\mathbf{N}}\right)\right|^{2} \mathrm{~d} s_{1} \mathrm{~d} s_{2} \mathrm{dx}_{\mathbf{3}} \ldots \mathrm{d} \mathbf{x}_{\mathbf{N}}
$$

where $\mathbf{x}_{\mathbf{i}}$ is the combined position-spin coordinate $\left(x_{i}=\left\{r_{i}, s_{i}\right\}\right)$ vector for electron $i$, and $\rho\left(\mathbf{r}_{1}, \mathbf{r}_{2}\right)$ is the so-called "spinless" pair density, which describes the probability of 
finding electrons at $\mathbf{r}_{1}$ and $\mathbf{r}_{2}$. Although the pair density greatly simplifies the problem for many electron systems, we are left with a 6-coordinate function, which makes for difficult interpretation as visual representation is not possible. However, one can manipulate the pair density in order to simplify the number of variables, while explicitly retaining electron pair information. One important electron pair property that can be considered in positionspace is $\mathbf{R}$, the absolute position of the electron pair centre-of-mass.

The extracule density, $E(\mathbf{R})$ can be obtained as

$$
E(\mathbf{R})=\int \rho\left(\mathbf{r}_{1}, \mathbf{r}_{2}\right) \delta\left(\mathbf{R}-\frac{\mathbf{r}_{1}+\mathbf{r}_{2}}{2}\right) \mathrm{d} \mathbf{r}_{1} \mathrm{~d} \mathbf{r}_{2}
$$

where $\delta(\boldsymbol{x})$ is the 3-dimensional Dirac delta function. In this instance, we chose to explore the richer topology of the vectorized density, as understanding where the centre-of-mass of a particular localized electron pair is in 3-Dimensional space is far more insightful than the scalar analogue.

In the present work, the centre-of-mass of the electron pair will simply be referred to as the centre-of mass, not to be confused with the nuclear centre-of-mass of the entire molecule. Coleman first described the extracule density in 1967,[4] but the first published report involving calculations of $E(\mathbf{R})$ did not appear until the early 1980s.[5] In their seminal paper on the extracule density, Thakkar and Moore stated:

$E(\mathbf{R})$ deals with all the $N(N-1) / 2$ electron pairs present in a $N$-electron molecule, but the chemist usually is interested only in $N / 2$ 'chemical pairs'. [...] For RHF wavefunctions of systems with an even number of electrons, perhaps the 'chemical NSGs' can simply be taken to be $\operatorname{det}\left|\phi_{i} \alpha \phi_{i} \beta\right|$ where the $\phi_{i}$ are localized molecular orbitals defined in one of the usual ways.

While this statement was made in the context of extracules, it applies equally to any form of electron pair analysis. This approach of analyzing electronic structure with respect to localized molecular orbitals was first explored in our lab using the intracule density.[6, 7]. More recently, we expanded this localized pair model of electronic structure analysis to include extracules. [8] Localized molecular orbitals (LMOs) are chemically intuitive in that they tend to represent covalent bonds and lone pairs quite well while retaining invariance from canonical molecular orbitals via a unitary transformation. By using LMOs, we can directly analyze the electron pair behaviour in molecular systems, and specifically the 
hydrogen bonding interaction (i.e. the $\sigma_{X H}$ and $n_{Y}$ orbitals) within the present work to observe how the centre-of-mass of the electrons described by these LMOs changes as the hydrogen bonding complex forms. Subsequently, we may also explore how such a density differs within different hydrogen bonding environments.

There are numerous techniques which have been developed for obtaining these localized molecular orbitals from their canonical counterparts.[9-12] The concept of LMOs was first proposed by Lennard-Jones and Pople in the early 1950s. The actual calculations of such orbitals didn't occur until the 1960s when Foster and Boys developed the first localization scheme based on maximizing the distance between centroids of charge and Edmiston and Ruedenberg developed a separate localization algorithm to maximize intraorbital repulsion (thereby minimizing exchange repulsion between individual LMOs).[9, 10] While both models remain popular for the determination of LMOs, the Edmiston-Ruedenberg (ER) model is more robust for our purposes, despite being more computationally intensive. For this reason the work presented herein is based on the ER localization scheme. While our focus in the present report is to describe electron pair distributions within LMOs, the localized pair model is capable of calculating intracule/extracule densities for any type of orbital including canonical molecular orbitals, absolutely localized molecular orbitals, [13-18] intrinsic bond orbitals, $[19,20]$ and natural bond orbitals (NBOs)[21-25] as well as for full molecular systems.

\section{COMPUTATIONAL METHODS}

Localization algorithms can be applied to any single determinant method, such as Hartree-Fock theory, which we explored in previous work describing the use of extracules in localized space,[8] or as will be explored here, Kohn-Sham (KS) DFT. What remains to be seen is whether analyzing a property of electron pairs is feasible using a technique which only considers the one-electron density, $\rho(\mathbf{r})$. The traditional electron density is defined as

$$
\rho(\mathbf{r})=\int\left|\Psi\left(\mathbf{x}_{\mathbf{1}}, \mathbf{x}_{\mathbf{2}}, \ldots, \mathbf{x}_{\mathbf{N}}\right)\right|^{2} \mathrm{~d} s_{1} \mathrm{dx}_{\mathbf{2}} \ldots \mathrm{d} \mathbf{x}_{\mathbf{N}}
$$

A two-electron, single determinant wave function can be obtained from a single localized molecular orbital, $\psi_{k}$. Using this definition in place of the molecular wave function in equation (3), one obtains the spin-reduced two-electron density for the pair of electrons 
described by that specific LMO, $\rho^{k}\left(\mathbf{r}_{1}, \mathbf{r}_{2}\right)$. For the singlet state of a localized molecular orbital, no exchange interactions exist. In this scenario and defining the electron density for the specific LMO, $k$, as was done for $\rho^{k}\left(\mathbf{r}_{1}, \mathbf{r}_{2}\right)$, the localized pair density is given by:

$$
\rho^{k}\left(\mathbf{r}_{\mathbf{1}}, \mathbf{r}_{\mathbf{2}}\right)=\rho^{k}\left(\mathbf{r}_{\mathbf{1}}\right) \rho^{k}\left(\mathbf{r}_{\mathbf{2}}\right)
$$

In doing so, Coulombic electron correlation effects are being ignored. This omission is implicitly accounted for to a certain extent through the correlation functional, but it is still an approximation.

Substituting (4) into (2) yields

$$
E(\mathbf{R})=\int \rho^{k}(\mathbf{r}) \rho^{k}(2 \mathbf{R}-\mathbf{r}) \mathrm{d} \mathbf{r}
$$

If we define the molecular orbitals as a linear combination of Gaussian primitives, we obtain:

$$
E(\mathbf{R})=\sum_{\mu \nu \lambda \sigma}^{K} \Gamma_{\mu \nu \lambda \sigma}^{\mathrm{KS}}(\mu \nu \lambda \sigma)_{E}
$$

where $(\mu \nu \lambda \sigma)_{E}$ denotes the extracule integrals evaluated for the Gaussian primitives $\mu, \nu$, $\lambda$, and $\sigma$ and $\Gamma_{\mu \nu \lambda \sigma}^{\mathrm{KS}}$ is the two-particle density matrix. These integrals are given by

$$
(\mu \nu \lambda \sigma)_{E}=\int \phi_{\mu}^{*}(\mathbf{r}) \phi_{\nu}(\mathbf{r}) \phi_{\lambda}^{*}(2 \mathbf{R}-\mathbf{r}) \phi_{\sigma}(2 \mathbf{R}-\mathbf{r}) \mathrm{d} \mathbf{r}
$$

where $\phi_{i}$ describes the $i^{t h}$ basis function. Thakkar and Moore developed a series of closed form expressions for these extracule integrals for Gaussian primitives of arbitrary angular momenta.[5] When our basis set is comprised of Gaussian primitives which are described by

$$
\phi(\mathbf{r})=\left(r_{x}-A_{x}\right)^{l}\left(r_{y}-A_{y}\right)^{m}\left(r_{z}-A_{z}\right)^{n} e^{-\alpha|\mathbf{r}-\mathbf{A}|^{2}}
$$

where $\alpha$ denotes the Gaussian exponent and $\mathbf{A}=\left\{A_{x}, A_{y}, A_{z}\right\}$ describes the center of the function, the general expression for these integrals is

$$
\begin{aligned}
(\mu \nu \lambda \sigma)_{E}=\left(\frac{4 \pi}{\zeta+\eta}\right)^{3 / 2} \exp [ & \left.-\frac{\zeta \eta(2 \mathbf{R}-\mathbf{P}-\mathbf{Q})^{2}}{\zeta+\eta}\right] \\
& \times \exp \left[-\frac{\alpha \beta(\mathbf{A}-\mathbf{B})^{2}}{\zeta}-\frac{\gamma \delta(\mathbf{C}-\mathbf{D})^{2}}{\eta}\right] T_{x} T_{y} T_{z}
\end{aligned}
$$

Here, $\alpha, \beta, \gamma$, and $\delta$ are the exponents of the four Gaussian primitives which are centered at $\mathbf{A}, \mathbf{B}, \mathbf{C}$, and $\mathbf{D}$, respectively. The variables $\zeta$ and $\eta$ are collections of exponents with 
$\zeta=\alpha+\beta$ and $\eta=\gamma+\delta$ while $\mathbf{P}$ and $\mathbf{Q}$ are defined as follows:

$$
\begin{aligned}
& \mathbf{P}=\frac{\alpha \mathbf{A}+\beta \mathbf{B}}{\zeta} \\
& \mathbf{Q}=\frac{\gamma \mathbf{C}+\delta \mathbf{D}}{\eta}
\end{aligned}
$$

The remaining variables, $T_{i}$ are angular factors (in the $x, y$, and $z$ directions) which account for differences in the integrals due to the angular momenta of the basis functions. For a set of $s$-type Gaussians, these factors are equal to unity. Here, we will simply define $T_{x}$; however, $T_{y}$ and $T_{z}$ can be obtained in an analogous fashion using the respective components for those Cartesian directions. $T_{x}$ is given by

$$
\begin{aligned}
T_{x}=\sum_{i=0}^{l_{\mu}+l_{\nu}} \Lambda_{i}\left(l_{\mu}, l_{\nu},\right. & \left.P_{x}-A_{x}, P_{x}-B_{x}, \zeta\right) \\
& \times \sum_{j=0}^{l_{\lambda}+l_{\sigma}} s_{i+j} \Lambda_{j}\left(l_{\lambda}, l_{\sigma}, Q_{x}-C_{x}, Q_{x}-D_{x}, \eta\right)
\end{aligned}
$$

Each of the angular factors contain $s_{k}$ which is defined below along with the functional forms of its two components, $g_{k}$ and $h_{k}$ :

$$
\begin{aligned}
s_{k} & =\frac{1}{g_{k}} \sum_{j=0}^{k / 2} g_{j} h_{k-2 j} \\
h_{k} & =\left(P_{x}+Q_{x}-2 R_{x}\right)^{k} \times \frac{1}{k !} \\
g_{k} & =\left(\frac{\zeta+\eta}{4 \zeta \eta}\right)^{k} \times \frac{1}{k !}
\end{aligned}
$$

where $0 \leq k \leq l_{\mu}+l_{\nu}+l_{\lambda}+l_{\sigma}$ in which $l_{i}$ denotes the angular momentum of gaussian primitive $i$, in the $x$-axis. The other primary component of the $T_{i}$ is the $\Lambda$ function which is defined by:

$$
\Lambda_{j}\left(l_{1}, l_{2}, a, b, c\right)=\sum_{k=0}^{\left(l_{1}+l_{2}-j\right) / 2} f_{2 k+j}\left(l_{1}, l_{2}, a, b\right) \frac{(2 k+j) !}{(4 c)^{k+j} k ! j !}
$$

Within this expression, $f_{j}$ is an integer defined by the polynomial coefficients of:

$$
\sum_{j=0}^{l_{1}+l_{2}} f_{j}\left(l_{1}, l_{2}, a, b\right) x^{j}=(x+a)^{l_{1}}(x+b)^{l_{2}}
$$

The value of the extracule density was calculated at various points defined by the MuraKnowles grid that was adapted to extend in the negative directions of the Cartesian coordinates. The grid was overlaid on the $y z$-plane and consisted of 151 points (75 in each 
direction as well as the origin). For the purposes of this study, the two atoms involved in the bond $(\mathrm{X}-\mathrm{H})$ containing the hydrogen atom as well as the atom containing the lone pair $(\mathrm{Y})$ that will participate in hydrogen bonding are all positioned in the $y z$-plane to ensure adequate sampling of the environments around these atoms.

To quantify the extracules, there were a number of metrics employed. Since slices were chosen as opposed to all of 3-D space, the extracule did not integrate to unity (i.e. number of electron pairs; a necessary condition for single orbitals), and instead is equal to the value of $E\left(\boldsymbol{R}_{y z}\right)$ integrated over the full slice. We define this metric as the zeroth moment, $\left\langle\boldsymbol{R}_{\boldsymbol{y} z}^{\mathbf{0}}\right\rangle$, which is defined as

$$
\left\langle\boldsymbol{R}_{\boldsymbol{y z}}^{\mathbf{0}}\right\rangle=\int_{-\infty}^{\infty} \int_{-\infty}^{\infty} E\left(\boldsymbol{R}_{\boldsymbol{y} z}\right) \mathrm{d} R_{y} \mathrm{~d} R_{z}
$$

Similarly, we can look at the first moment of $R_{z}$ to determine the average value in the bond axis direction ( $z$-axis). This is evaluated as

$$
\left\langle R_{z}\right\rangle=\frac{1}{\left\langle\boldsymbol{R}_{\boldsymbol{y} \boldsymbol{z}}^{\mathbf{0}}\right\rangle} \int_{-\infty}^{\infty} \int_{-\infty}^{\infty} R_{z} \times E\left(\boldsymbol{R}_{\boldsymbol{y} \boldsymbol{z}}\right) \mathrm{d} R_{y} \mathrm{~d} R_{z}
$$

The final quantifiable property of interest in this study is the position of the extracule density maximum in the bond axis, $R_{z}^{\max }$.

Each of these properties can be calculated for a hydrogen bonding complex without needing the extracule density for either the $\mathrm{X}-\mathrm{H}$ or $\mathrm{Y}$ molecule by itself. However, one can imagine that comparing these complexes to the molecules in the absence of the hydrogen bonding interaction would be rather insightful. To do this, we explore the extracule deformation density, $\Delta E_{d}^{\phi}(\mathbf{R})$, for orbital $\phi$ which is defined as

$$
\Delta E_{d}^{\phi}(\mathbf{R})=E_{d}^{\phi, \text { complex }}(\mathbf{R})-E^{\phi, \text { molecule }}(\mathbf{R})
$$

The deformation density is useful in demonstrating the effect on the extracule density as the hydrogen bonding interaction forms and how it changes as the distance between the two molecules increases. The main property of interest involving the deformation density is $\delta_{R}$ which is the magnitude of the difference between the complex and single molecule. This is defined as

$$
\delta_{R}=\int_{-\infty}^{\infty} \int_{-\infty}^{\infty}\left|\Delta_{d}^{\phi} E\left(\boldsymbol{R}_{y z}\right)\right| \mathrm{d} R_{y} \mathrm{~d} R_{z}
$$

All geometries for the hydrogen bonding complexes were obtained from Hobza's X40x10 and S66x8 data sets.[26, 27] Single-point energy and orbital localization calculations were performed on each of these geometries at the M06-2X/u6-311G(d,p) level of 
theory ( $\mathrm{u}$ indicates that the basis set was completely uncontracted) using the GAMESS software package.[28] The Mathematica 8 software package was utilized to interpolate the data obtained from grid-point evaluation for visual representation and further analysis. [29] Atomic units are used throughout unless otherwise stated.

Additional calculations utilizing the "Atoms in Molecules" (AIM) technique were carried out on these systems to compare the capabilities of extracules in the localized pair model to AIM theory with regards to interpreting hydrogen bonding interactions. Past research[3035] has shown the utility of AIM theory in the study of hydrogen bonding interactions and thus, it should represent a good case for comparing the results of our novel approach.

\section{RESULTS AND DISCUSSION}

For the purpose of this study, 28 hydrogen bonding complexes were chosen from the X40x10 and S66x8 data sets. [26, 27] For each of the systems the extracule, $E\left(\boldsymbol{R}_{\boldsymbol{y z}}\right)$, density was calculated for the $\sigma_{\mathrm{XH}} \mathrm{LMO}$ of the proton donor individually and in the $\mathrm{X}-\mathrm{H}^{\cdots \cdots} \mathrm{Y}$ hydrogen bonding complex at $\mathrm{H}^{\cdots} \mathrm{Y}$ separations of $0.90 d_{0}, 0.95 d_{0}, 1.00 d_{0}, 1.05 d_{0}, 1.10 d_{0}$, $1.25 d_{0}, 1.50 d_{0}$, and $2.00 d_{0}$, where $d_{0}$ is the geometry optimized distance between the two atoms (see Figure 1). Likewise, for $n_{\mathrm{Y}}, E\left(\boldsymbol{R}_{\boldsymbol{y z}}\right)$ was calculated for the complex at each of the separations listed above, as well as that for the isolated proton acceptor, Y.

$$
\stackrel{\mathrm{d}}{\mathrm{X}-\mathrm{H} \cdots \mathrm{Y}} \quad \mathrm{d}=0.90 \mathrm{~d}_{0}-2.00 \mathrm{~d}_{0}
$$

FIG. 1: Pictorial representation of the various geometries for each of the hydrogen bonding complexes.

\section{Extracule densities for $\sigma_{\mathrm{XH}}$ LMOs}

When a hydrogen bond forms, the $n_{\mathrm{Y}} \mathrm{LMO}$ interacts with the electron deficient $\mathrm{H}$ atom in the $\mathrm{X}-\mathrm{H}$ molecule. In doing so, one expects that the electron pair, and thus the centreof-mass, within the $\mathrm{X}-\mathrm{H}$ bond would migrate towards the $\mathrm{X}$ atom due to the donation of election density from the lone pair to the hydrogen. This effect can be observed through both $\left\langle R_{z}\right\rangle$ and $R_{z}^{\max }$. In the present molecules, the $\mathrm{X}-\mathrm{H}$ bond midpoint is placed at the 
TABLE I: $\left\langle\boldsymbol{R}_{y z}\right\rangle$ for the $\sigma_{O-H}$ in MeOH-Y complexes

\begin{tabular}{lccccccccc}
\hline System & $0.90 d_{0}$ & $0.95 d_{0}$ & $1.00 d_{0}$ & $1.05 d_{0}$ & $1.10 d_{0}$ & $1.25 d_{0}$ & $1.50 d_{0}$ & $2.00 d_{0}$ & $\infty$ \\
\hline $\mathrm{MeOH}-\mathrm{CH}_{3} \mathrm{Cl}$ & 0.0184 & 0.0223 & 0.0253 & 0.0275 & 0.0291 & 0.0319 & 0.0345 & 0.0359 & 0.0365 \\
$\mathrm{MeOH}-\mathrm{CH}_{3} \mathrm{~F}$ & 0.0102 & 0.0151 & 0.0190 & 0.0220 & 0.0244 & 0.0287 & 0.0324 & 0.0349 & 0.0365 \\
$\mathrm{MeOH}-\mathrm{MeNH}_{2}$ & -0.0278 & -0.0194 & -0.0123 & -0.0063 & -0.0012 & 0.0098 & 0.0195 & 0.0274 & 0.0323 \\
$\mathrm{MeOH}-\mathrm{MeOH}$ & 0.0043 & 0.0117 & 0.0178 & 0.0229 & 0.0271 & 0.0355 & 0.0424 & 0.0478 & 0.0511 \\
$\mathrm{MeOH}-\mathrm{Peptide}$ & -0.0091 & -0.0008 & 0.0061 & 0.0120 & 0.0170 & 0.0275 & 0.0375 & 0.0461 & 0.0495 \\
$\mathrm{MeOH}-\mathrm{Pyridine}$ & -0.0273 & -0.0184 & -0.0109 & -0.0027 & 0.0019 & 0.0121 & 0.0216 & 0.0283 & 0.0329 \\
$\mathrm{MeOH}-\mathrm{H}_{2} \mathrm{O}$ & 0.0044 & 0.0111 & 0.0166 & 0.0211 & 0.0247 & 0.0321 & 0.0384 & 0.0435 & 0.0469 \\
\hline
\end{tabular}

origin, with $\mathrm{X}$ in the negative $z$-direction and the hydrogen in the positive direction. The data presented in Table I clearly show that as $\mathrm{Y}$ approaches the $\mathrm{X}-\mathrm{H}$ molecule, the average value of $R_{z}$ shifts towards the $\mathrm{X}$ atom, as predicted. This effect is seen universally across the data set as shown in the table and in the Electronic Supplementary Information (ESI). The presence of the Y lone pair causes a small but significant migration of the centre-ofmass in the $\sigma_{\mathrm{X}-\mathrm{H}}$ bond towards the $\mathrm{X}$ atom. This effect is also observed in $R_{z}^{\max }$ which is included in the ESI.

In our previous work introducing the localized extracule, we noted a few trends in $\left\langle\boldsymbol{R}_{\boldsymbol{y} z}^{\mathbf{0}}\right\rangle$ [8]. First, the primary effect was that by introducing electronegative species to the neighboring chemical environment, increases in $\left\langle\boldsymbol{R}_{\boldsymbol{y} z}^{0}\right\rangle$ were observed in nearly all cases. This signifies that the value of the extracule density, $E(\mathbf{R})$, in the plane of the hydrogen bond increases as the electronegativity of neighboring substituents increases indicating a migration of the centre-of-mass density towards the bonding region. The only exceptions were observed in systems where a second row element was bonded to a first row element. A similar trend is observed for these hydrogen bonding systems. All systems exhibit an increase in $\left\langle\boldsymbol{R}_{\boldsymbol{y} \boldsymbol{z}}^{\mathbf{0}}\right\rangle$ as the proton acceptor $\mathrm{Y}$ is drawn closer to the donor species, with the exception of systems where the donor contains an $\mathrm{X}-\mathrm{H}$ bond with $\mathrm{X}=\mathrm{Cl}, \mathrm{Br}$.

Within the context of AIM theory, an electron density analysis technique, values of the electron density at bond critical points $\left(\rho\left(r_{c}\right)\right)$ have yielded direct relationships to hydrogen bond strengths. This led us to explore whether any of the metrics employed herein would show similar predictive capacity. Figure 2 depicts the relationship between hydro- 
TABLE II: $\left\langle\boldsymbol{R}_{\boldsymbol{y} \boldsymbol{z}}^{\mathbf{0}}\right\rangle$ for the $\sigma_{\mathrm{X}-\mathrm{H}}$ bond in a few select systems

\begin{tabular}{lccccccccc}
\hline System & $0.90 d_{0}$ & $0.95 d_{0}$ & $1.00 d_{0}$ & $1.05 d_{0}$ & $1.10 d_{0}$ & $1.25 d_{0}$ & $1.50 d_{0}$ & $2.00 d_{0}$ & $\infty$ \\
\hline $\mathrm{HBr}-\mathrm{MeOH}$ & 0.6620 & 0.6632 & 0.6640 & 0.6646 & 0.6651 & 0.6663 & 0.6674 & 0.6679 & 0.6682 \\
$\mathrm{HCl}-\mathrm{MeNH}_{2}$ & 0.7176 & 0.7191 & 0.7205 & 0.7217 & 0.7227 & 0.7247 & 0.7262 & 0.7272 & 0.7271 \\
$\mathrm{HCl}-\mathrm{MeOH}$ & 0.7340 & 0.7347 & 0.7351 & 0.7353 & 0.7356 & 0.7368 & 0.7368 & 0.7368 & 0.7368 \\
$\mathrm{HF}-\mathrm{MeNH}_{2}$ & 1.0749 & 1.0712 & 1.0681 & 1.0655 & 1.0631 & 1.0576 & 1.0526 & 1.0498 & 1.0480 \\
$\mathrm{HF}-\mathrm{MeOH}$ & 1.0724 & 1.0697 & 1.0673 & 1.0652 & 1.0634 & 1.0597 & 1.0572 & 1.0553 & 1.0541 \\
$\mathrm{MeNH}_{2}-\mathrm{MeNH}_{2}$ & 0.8417 & 0.8408 & 0.8401 & 0.8395 & 0.8391 & 0.8385 & 0.8380 & 0.8374 & 0.8371 \\
$\mathrm{MeNH}_{2}-\mathrm{MeOH}$ & 0.8413 & 0.8406 & 0.8401 & 0.8397 & 0.8394 & 0.8389 & 0.8384 & 0.8378 & 0.8375 \\
\hline
\end{tabular}

gen bond strengths to $\left\langle\boldsymbol{R}_{\boldsymbol{y} \boldsymbol{z}}^{\mathbf{0}}\right\rangle$ for each of the systems at equilibrium (i.e. $d=1.00 d_{0}$ ). While there does not appear to be any mathematical relationship, the graph clearly classifies the systems into 5 separate classes where the interaction energy may vary, but the zeroth moment is effectively unchanged. Each class corresponds to a particular X atom in the $\mathrm{X}-\mathrm{H}$ bond. In increasing order of $\left\langle\boldsymbol{R}_{\boldsymbol{y} z}^{0}\right\rangle$, these classes represent $\mathrm{Br}-\mathrm{H}, \mathrm{Cl}-\mathrm{H}, \mathrm{N}-\mathrm{H}, \mathrm{O}-\mathrm{H}$ and $\mathrm{F}-\mathrm{H}$ bonds. This ordering agrees with the previously noted relationship between $\left\langle\boldsymbol{R}_{\boldsymbol{y} \boldsymbol{z}}^{\mathbf{0}}\right\rangle$ and electronegativity. The lone system containing Br (the lowest electronegativity) falls at the lower end of $\left\langle\boldsymbol{R}_{\boldsymbol{y} \boldsymbol{z}}^{\mathbf{0}}\right\rangle$ values, while increases in electronegativity lead to an increase in the value of the metric.

The extracule deformation density is a useful visual tool as it highlights all of the trends that have been discussed thus far. Figure 3 illustrates $\Delta E_{1.00 d_{0}}^{\phi \text {,complex }}\left(\boldsymbol{R}_{\boldsymbol{y} z}\right)$ for the $\sigma_{\mathrm{N}-\mathrm{H}}$ bond and $n_{\mathrm{Y}} \mathrm{LMOs}$ in the $\mathrm{MeNH}_{2}-\mathrm{MeOH}$ hydrogen bonding complex at its equilibrium geometry. This figure clearly demonstrates that the centre-of-mass in the $\sigma_{\mathrm{N}-\mathrm{H}}$ bond migrates away from the hydrogen atom towards the $\mathrm{X}$ atom. This migration is caused by the shift in the centre-of-mass from the lone pair, $n_{\mathrm{O}}$ towards the hydrogen in X-H during the formation of the hydrogen bond. This agrees with the previous observation that both $\left\langle R_{z}\right\rangle$ and $R_{z}^{\max }$ shift towards $\mathrm{X}$ when the hydrogen bond forms and as the hydrogen bonding distance decreases.

In comparing Figures 4(a) and (b), it is noted that as the two molecules involved in the hydrogen bonding interaction separate, the extracule of the complex begins to resemble that of the single molecule in the absence of hydrogen bonding. The degree to which the 


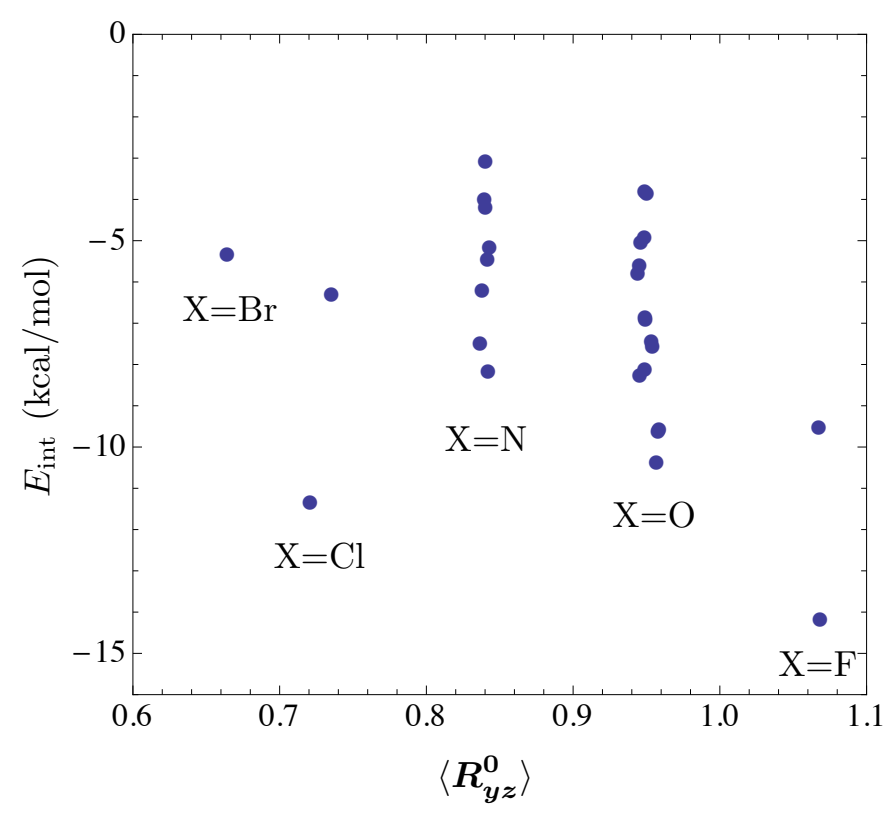

FIG. 2: Classification of hydrogen bonding systems based on $\left\langle\boldsymbol{R}_{\boldsymbol{y} \boldsymbol{z}}^{\mathbf{0}}\right\rangle$ of the $\sigma_{\mathrm{X}-\mathrm{H}}$ LMO and hydrogen bonding strengths.

extracules differ can be measured using $\delta_{R}$. This property is tabulated in Table III for the complexes where water is the donor species (data for all systems can be found in the ESI). These data clearly show that a greater difference in the extracule density is observed when the two species are in close proximity. This difference tails off significantly as the systems reach a separation of $2.00 d_{0}$. This again suggests a propensity for the localized extracule to offer, at minimum, qualitative insight into the strengths of the non-covalent interactions.

For a given complex, analysis of each quantitative metric described in the computational section demonstrates a strong quadratic relationship with respect to interaction energy

TABLE III: $\delta_{R}$ for $\phi=\sigma_{\mathrm{O}-\mathrm{H}}$ in the $\mathrm{H}_{2} \mathrm{O}-\mathrm{Y}$ subset of systems.

\begin{tabular}{lllllllll}
\hline System & $0.90 d_{0}$ & $0.95 d_{0}$ & $1.00 d_{0}$ & $1.05 d_{0}$ & $1.10 d_{0}$ & $1.25 d_{0}$ & $1.50 d_{0}$ & $2.00 d_{0}$ \\
\hline $\mathrm{H}_{2} \mathrm{O}-\mathrm{MeNH}_{2}$ & 0.0866 & 0.0735 & 0.0626 & 0.0535 & 0.0459 & 0.0298 & 0.0162 & 0.0060 \\
$\mathrm{H}_{2} \mathrm{O}-\mathrm{MeOH}$ & 0.0677 & 0.0564 & 0.0472 & 0.0397 & 0.0336 & 0.0213 & 0.0114 & 0.0043 \\
$\mathrm{H}_{2} \mathrm{O}-\mathrm{Peptide}$ & 0.1205 & 0.1109 & 0.1036 & 0.0981 & 0.0938 & 0.0860 & 0.0811 & 0.0795 \\
$\mathrm{H}_{2} \mathrm{O}-\mathrm{Pyridine}$ & 0.1156 & 0.1057 & 0.0983 & 0.0927 & 0.0885 & 0.0812 & 0.0772 & 0.0762 \\
$\mathrm{H}_{2} \mathrm{O}-\mathrm{H}_{2} \mathrm{O}$ & 0.0149 & 0.0137 & 0.0108 & 0.0093 & 0.0081 & 0.0055 & 0.0031 & 0.0011 \\
\hline
\end{tabular}



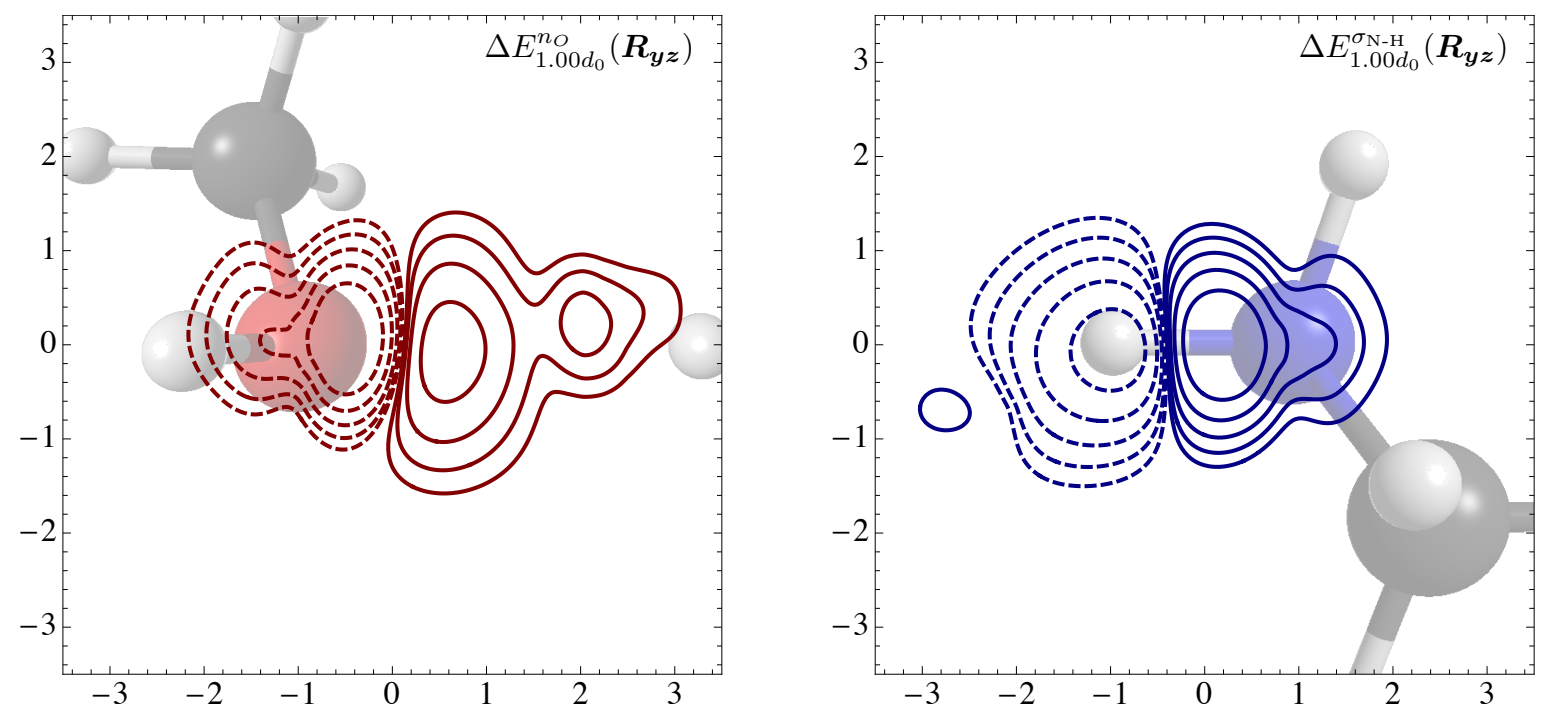

FIG. 3: Contour plot of $\Delta E_{1.00 d_{0}}^{\sigma_{\mathrm{N}-\mathrm{H}}}\left(\boldsymbol{R}_{\boldsymbol{y} \boldsymbol{z}}\right)$ and $\Delta E_{1.00 d_{0}}^{n_{\mathrm{O}}}\left(\boldsymbol{R}_{\boldsymbol{y} \boldsymbol{z}}\right)$ in $\mathrm{MeNH}_{2}-\mathrm{MeOH}$ depicting the change in the extracule density in the presence of the proton acceptor, $\mathrm{MeOH}$ (dashed lines signify negative contours while solid lines signify the positive contours).

(Figure 5). While it is expected that this relationship would not hold for larger separations, encountering such interactions in real chemical systems would be rare. Over the 28 systems analyzed, coefficients of determination $\left(R^{2}\right)$ values ranged from 0.9113-0.9999. However, with the exceptions of $\mathrm{H}_{2} \mathrm{O}$-Pyridine $\left(\mathrm{R}^{2}=0.9113\right), \mathrm{H}_{2} \mathrm{O}$-Peptide $\left(\mathrm{R}^{2}=0.9257\right)$, and Peptide- $\mathrm{H}_{2} \mathrm{O}\left(\mathrm{R}^{2}=0.9717\right)$ complexes, all other systems had $\mathrm{R}^{2}$ values greater than or equal to 0.9941 . In the case of the first two complexes, there may be some other form of interaction that is occurring in these systems that is causing these deviations from the quadratic relationship while the fit of the third appears to be distorted by a single data point.

As in the case of $\left\langle\boldsymbol{R}_{\boldsymbol{y} z}^{\mathbf{0}}\right\rangle$, we also explored the possibility of a relationship existing between $\delta_{R}$ for each of the systems when $d=1.00 d_{0}$. This relationship is depicted in Figure 6. The two points in red represent the $\mathrm{H}_{2} \mathrm{O}$-Pyridine and $\mathrm{H}_{2} \mathrm{O}$-Peptide complexes. When these two points are removed, the coefficient of determination improved from 0.7137 to 0.8411 . While this may not appear to have much predictive power, it is on par with bondcritical-point $(B C P)$ density analysis of AIM theory $\left(R^{2}=0.8282\right)$. Figure $\left.6 \mathrm{~b}\right)$ demonstrates this analysis. Unlike the case of the extracules, there are no distinct outliers in this analysis that could lead to an improvement in the $\mathrm{R}^{2}$ value. Past studies have shown that $\mathrm{BCP}$ den- 

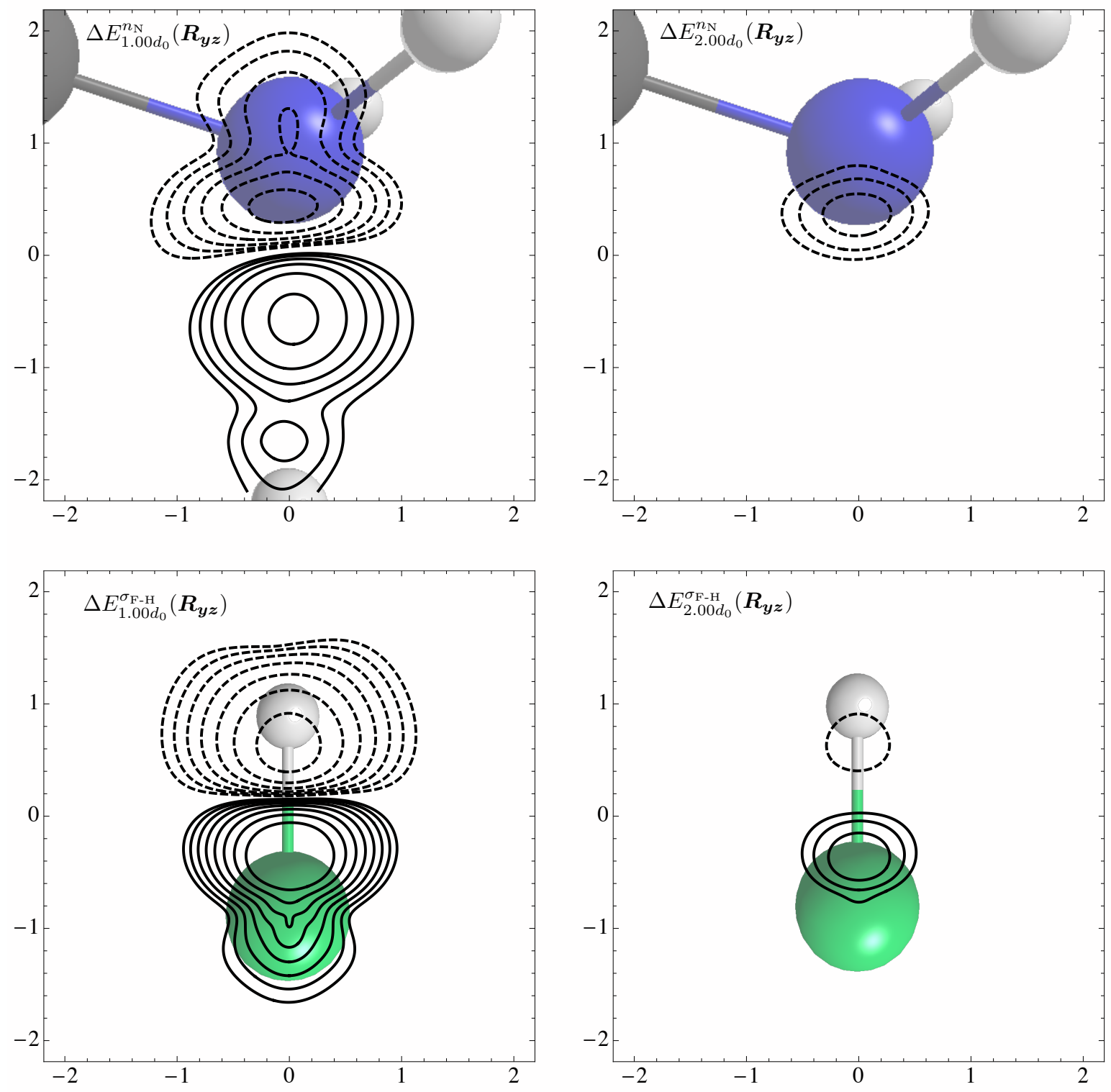

FIG. 4: Contour plot of $\Delta E_{d}^{\sigma_{\mathrm{F}-\mathrm{H}}}\left(\boldsymbol{R}_{\boldsymbol{y} \boldsymbol{z}}\right)$ and $\Delta E_{d}^{n_{\mathrm{N}}}\left(\boldsymbol{R}_{\boldsymbol{y} \boldsymbol{z}}\right)$ for the HF-MeNH $\mathrm{H}_{2}$ complex demonstrating the diminishing effect on $\Delta E(\mathbf{R})$ as the complex separates (dashed lines signify negative contours while solid lines signify the positive contours).

sities are strong predictors for hydrogen bonding[30-35] suggesting that the variability in this data set makes predictions rather difficult. The fact that our technique performs as well as it does for this data set is a strong indicator of its potential in the analysis of hydrogen bonded complexes and non-covalent interactions in general.

It should be noted that analogous studies were carried out on the $n_{Y}$ LMOs; however, these localized pairs proved to be far less predictive ann thus we conclude that it is the 

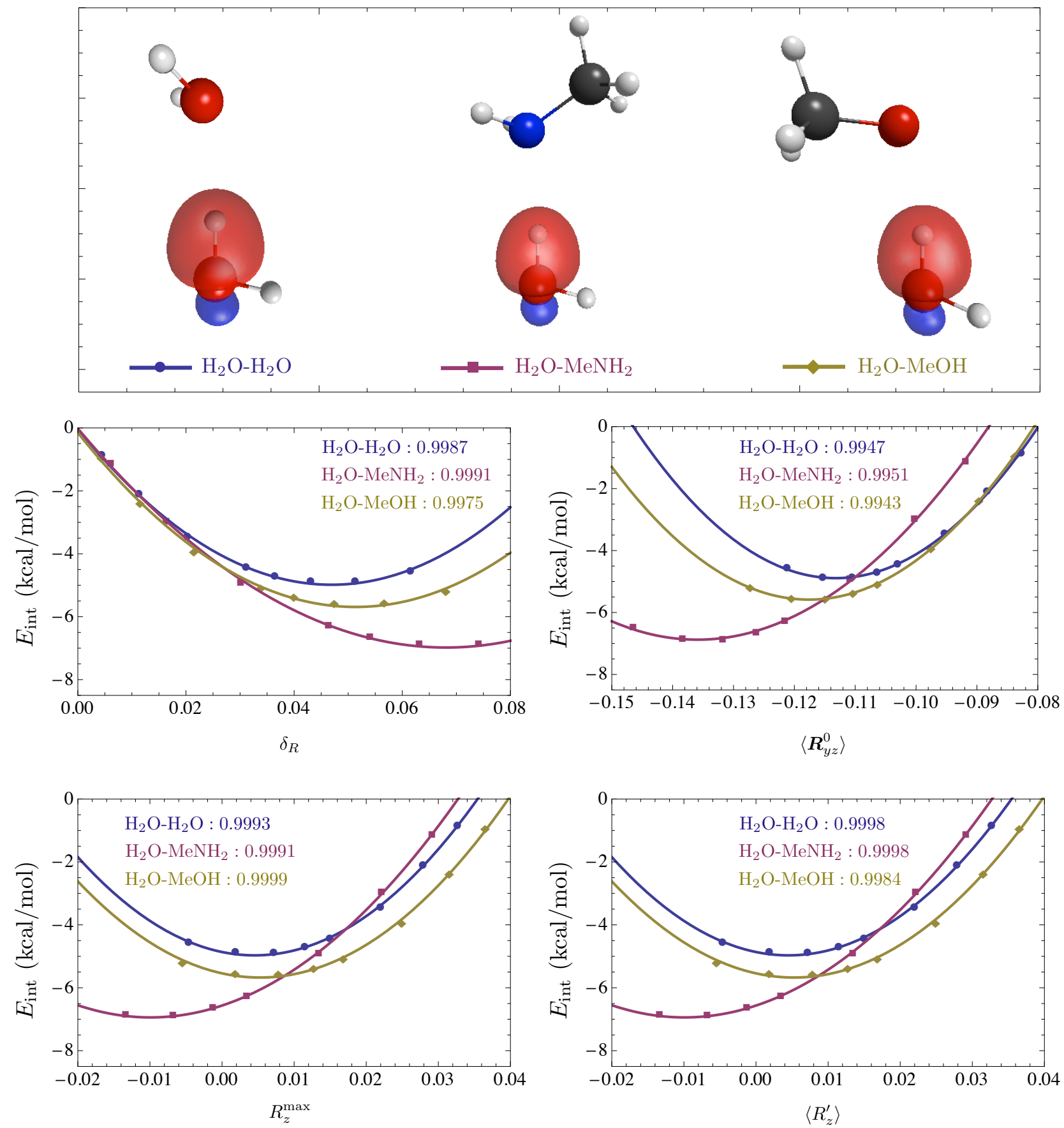

FIG. 5: Relationship between $\delta_{R},\left\langle\boldsymbol{R}_{\boldsymbol{y} z}^{0}\right\rangle, R_{z}^{\max }$, and $\left\langle R_{z}^{\prime}\right\rangle$ versus hydrogen bond strength for $\mathrm{H}_{2} \mathrm{O}$ $\mathrm{H}_{2} \mathrm{O}, \mathrm{H}_{2} \mathrm{O}-\mathrm{MeNH}_{2}$, and $\mathrm{H}_{2} \mathrm{O}-\mathrm{MeOH}$.

$\sigma_{\mathrm{X}-\mathrm{H}}$ bond that is most important when characterizing the hydrogen bonding interactions. All the data regarding the extracules for the lone pairs is supplied in the ESI. 

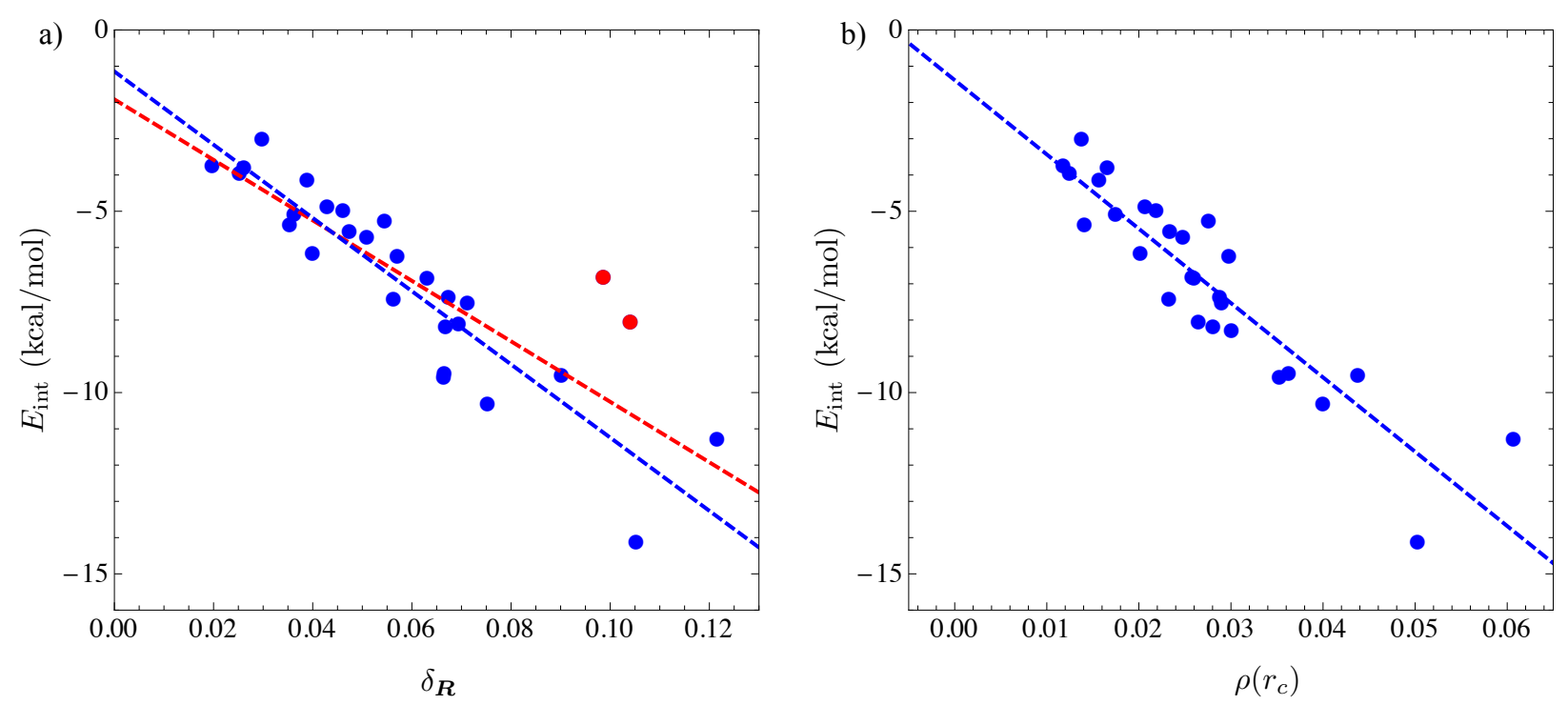

FIG. 6: Relationship between hydrogen bond interaction strength, $E_{\text {int }}$ versus a) $\delta_{R}$, and b) the density at the bond critical point, $\rho\left(r_{c}\right)$, for all systems at equilibrium.

\section{Scaling Metrics}

Of the four metrics discussed herein, the one which exhibited the strongest correlation to interaction energies was $\delta_{R}$. Considering this metric is the only one that explicitly incorporates the change in the electron pair upon hydrogen bond formation, it was decided that a similar approach would be applied to the other 3 metrics. Thus, the change in each metric was observed with the calculation of the change determined as follows:

$$
\Delta x=x^{\mathrm{X}-\mathrm{H} \cdots \mathrm{Y}}-x^{\mathrm{X}-\mathrm{H}}
$$

Somewhat surprisingly, this did not have a major effect on the correlations between any of the three metrics and $E_{\text {int }}$. An alternative scaling to the metrics was considered instead. In this case, the metrics were divided by the separation distance between the two species in the complex, $d_{0}$. While this did lead to improvements in relationships in most cases, $\delta_{R}$ remained the only metric with a strong relationship to interaction energies. Nonetheless, it did lead to a small, but significant improvement in the coefficient of determination for $\delta_{R}$ and $E_{\text {int }}$. By scaling each of the $\delta_{R}$ values by the hydrogen bond distance, the $\mathrm{R}^{2}$ value improved to 0.8954 from 0.8411 and yielded the following relationship

$$
E_{\text {int }}=-111.3 \frac{\delta_{R}}{d_{0}}-0.714
$$


While this may be considered a small improvement, it does improve upon an already strong relationship.

\section{CONCLUSIONS}

A novel method for the analysis of non-covalent interactions involving electron pairs, more specifically hydrogen bonding, was explored. This localized electron pair approach affords a quantitative analysis technique in accord with the resonance-covalency model as a characterization tool for hydrogen bonding and general non-covalent interactions. A clear migration of the centre-of-mass of localized electron pairs was consistently observed towards the hydrogen with respect to the $n_{Y}$ lone pair and away from the hydrogen in the case of the $\sigma_{X-H}$ bond. Strong quadratic relationships were observed between each of the studied metrics and interaction energies for a given hydrogen bonding complex indicating a unique predictive quality of our characterization.

When considering the entire set of molecules, a thorough study was conducted on each of the systems at their equilibrium geometries. Of the 4 metrics employed, $\delta_{R}$ demonstrated a strong relationship with respect to hydrogen bond strengths. This predictive power could be improved by studying the entire extracule density, as opposed to the single slices in the bond plane that were utilized here. The only issue with using $\delta_{R}$ as a predictive tool is that the extracule for the $\mathrm{X}-\mathrm{H}$ molecule in the absence of the proton acceptor, $\mathrm{Y}$, is required. As such, this tool would not be of any use for assessing intramolecular hydrogen bond strengths, but could still yield interesting topological information regarding the behaviour of the electron pair.

\section{ACKNOWLEDGEMENTS}

The authors would like to thank the Canadian Foundation for Innovation (CFI), the Natural Sciences and Engineering Research Council of Canada, and the University of Prince Edward Island for the financial support that made this research possible. Computational resources were provided by ACENET, WestGrid, and Compute Canada Calcul Canada. ACENET is funded by CFI, the Atlantic Canada Opportunities Agency (ACOA), and the provinces of Newfoundland and Labrador, Nova Scotia, and New Brunswick. 


\section{SUPPLEMENTARY INFORMATION}

All data points used in the analysis presented in this paper are provided in the supplementary information.

* E-mail:jpearson@upei.ca

[1] L. Pauling, The Nature of the Chemical Bond, Cornell University Press, Ithaca, 1939.

[2] F. Weinhold and R. A. Klein, Mol. Phys., 2012, 110, 565.

[3] F. Weinhold and R. A. Klein, Chem. Educ. Res. Pract., 2014, 15, 276.

[4] A. J. Coleman, Int. J. Quantum Chem., 1967, 1, 457.

[5] A. J. Thakkar and N. J. Moore, Int. J. Quantum Chem., 1981, 20, 393-400.

[6] Z. A. M. Zielinski and J. K. Pearson, Comp. Theor. Chem., 2013, 1003, 79-90.

[7] D. C. Hennessey, B. J. H. Sheppard, D. E. C. K. Mackenzie and J. K. Pearson, Phys. Chem. Chem. Phys., 2014, 16, 25548.

[8] A. J. Proud, D. E. C. K. Mackenzie and J. K. Pearson, Phys. Chem. Chem. Phys., 2015, 17, 20194.

[9] J. Foster and S. Boys, Rev. Mod. Phys., 1960, 32, 300-302.

[10] C. Edmiston and K. Ruedenberg, Rev. Mod. Phys., 1963, 35, 457-464.

[11] J. Pipek and P. Mezey, J. Chem. Phys., 1989, 90, 4916-4926.

[12] W. J. V. Niessen, J. Chem. Phys., 56, 1972, 4290.

[13] H. Stoll, G. Wagenblast and H. Preuss, Theor. Chem. Acta, 1980, 57, 169-178.

[14] J. M. Cullen, Int. J. Quantum Chem., Quantum Chem. Symp., 1991, 25, 193-212.

[15] E. Gianinetti, M. Raimondi and E. Tornaghi, Int. J. Quantum Chem., 1996, 60, 157-166.

[16] T. Nagata, O. Takahashi, K. Saito and S. Iwata, J. Chem. Phys., 2001, 115, 3553-3560.

[17] R. Z. Khaliullin, E. A. Cobar, R. C. Lochan, A. T. Bell and M. Head-Gordon, J. Phys. Chem. A, 2007, 111, 8753-8765.

[18] R. Z. Khaliullin, M. Head-Gordon and A. T. Bell, J. Chem. Phys., 2006, 124, 204105.

[19] G. Knizia, J. Chem. Theory Comput., 2013, 9, 4834.

[20] G. Knizia and J. E. M. N. Klein, Angew. Chem. Int. Ed., 2015, 54, 1.

[21] J. P. Foster and F. Weinhold, J. Am. Chem. Soc., 1980, 102, 7211-7218. 
[22] A. E. Reed, L. A. Curtiss and F. Weinhold, Chem. Rev., 1988, 88, 899-926.

[23] F. Weinhold and J. E. Carpenter, The Structure of Small Molecules and Ions, Plenum, New York, 1988, pp. 227-236.

[24] E. D. Glendening, C. R. Landis and F. Weinhold, WIREs Comput. Mol. Sci., 2012, 2, 1-42.

[25] F. Weinhold, J. Comp. Chem., 2012, 33, 2363-2379.

[26] J. Řezáč, K. E. Riley and P. Hobza, J. Chem. Theory Comput., 2011, 7, 2427.

[27] J. ez, K. E. Riley and P. Hobza, J. Chem. Theor. Comput., 2012, 8, 4285-4292.

[28] M. W. Schmidt, K. K. Baldridge, J. A. Boatz, S. T. Elbery, M. S. Gordon, J. H. Jensen, S. Koseki, N. Matsunaga, K. A. Nguyen, S. J. Su, T. L. Windus, M. Dupuis and J. A. Montgomery, J. Comput. Chem., 1993, 14, 1347-1363.

[29] Wolfram Research, Inc., Mathematica 8, 2010.

[30] P. L. A. Popelier, J. Phys. Chem. A, 1998, 102, 1873.

[31] S. J. Grabowski, J. Phys. Chem. A, 2001, 105, 10739.

[32] S. Wojtulewski and S. J. Grabowski, J. Chem. Phys., 2005, 309, 183.

[33] W. Zierkiewicz, P. Jurecka and P. Hobza, Chem. Phys. Chem., 2005, 6, 609.

[34] R. W. Gora, S. J. Grabowski and J. Leszczynski, J. Phys. Chem. A, 2005, 109, 6397.

[35] R. Parthasarathi, V. Subramanian and N. Sathyamurthy, J. Phys. Chem. A Lett., 2006, 110, 3349. 